\title{
Direct growth of hierarchically structured titanate nanotube filtration membrane for removal of waterborne pathogens
}

\author{
Haimin Zhang, Huijun Zhao*, Porun Liu, Shanqing Zhang, Guiying Li \\ Australian Rivers Institute and Griffith School of Environment, Gold Coast Campus, Griffith University, \\ Queensland 4222, Australia
}

* Corresponding author. Tel.: +61 7 55528261. Fax: +61 7 55528067. E-mail: h.zhao@griffith.edu.au (H.J. Zhao.). 


\section{ABSTRACT}

A hierarchical titanate nanotube based filtration membrane was fabricated and successfully applied for bacteria removal. A facile and effective membrane fabrication method was developed to directly grow a hierarchical titanate nanotube selective layer onto a porous metal membrane substrate. The method is a one-pot synthesis method, eliminates the needs for tedious and costly multiple-coating approach. The resultant membrane possesses a unique porous structure with strong mechanical strength, intrinsically free of cracks and pinholes, intrinsically free of cracks and pinholes and can be readily regenerated by a simple pressure driven back-flushing process. Successful separation of E. coli demonstrates the applicability of the titanate nanotube membrane for waterborne pathogens removal, which would be of a great interest to the water purification applications, especially for the purified recycling water applications. The high selectivity and flux of the nanotube membrane in addition to its excellent biocompatibility and nontoxic nature make such a membrane highly attractive to medical applications for removal pathogens and other unwanted biological constituents with sizes greater than $50 \mathrm{~nm}$ from highly complex medium.

Keywords: Titanate nanotube membrane; Hydrothermal synthesis; Waterborne pathogens removal; Membrane separation 


\section{Introduction}

Inorganic membranes have been a hot topic of new membrane technology development in the past two decades [1-5]. Among them, nanostructured $\mathrm{TiO}_{2}$ based membranes have become a central focus of this new generation inorganic membranes in the recent decade, attracting enormous research activities and commercial interests [5-9]. These are mainly due to the superior chemical, mechanical and thermal stability of $\mathrm{TiO}_{2}$ and its suitability for constructing membranes with a wide range of pore sizes for different applications [5, 6, 8, 10]. Since Anderson et al's pioneering work in 1988 [11], extensive research activities have been conducted to fabricate $\mathrm{TiO}_{2}$ based membranes for various applications covering dairy, beverage, food, pharmaceutical, chemical, biochemical, electronic and water/wastewater treatment industries $[1-3,5,6,8,10,12]$. In addition, a number of recent reports also revealed some interesting advantages of $\mathrm{TiO}_{2}$ based membranes [13-16]. Albu et al [13] and Zhang et al [14] demonstrated that the photocatalytic functions of the $\mathrm{TiO}_{2}$ can be utilised in a membrane separation process. These membrane systems, on one hand, maintain its size-selective based separation ability, on the other hand, are capable of simultaneously remove unwanted pollutants by means of photocatalytic decomposition. Sang and co-workers $[15,16]$ reported the use of $\mathrm{TiO}_{2}$ surface absorption properties to remove heavy metal ions. These studies displayed very promising membrane performance improvement by utilising chemical and photochemical properties of $\mathrm{TiO}_{2}$.

To date, $\mathrm{TiO}_{2}$ based membranes were fabricated with variety of methods including sol-gel, hydrothermal, chemical vapor deposition, plasma-enhanced chemical vapor deposition, anodic oxidation, and slip casting methods. Despite these varieties, all $\mathrm{TiO}_{2}$ membranes share a common asymmetric structural feature - a $\mathrm{TiO}_{2}$ dense layer (responsible for selectivity) forms on the top of a porous membrane substrate (i.e. porous stainless steel, alumina or silica, responsible for providing mechanical and other supports) $[5,12,14,15,17]$. In order to obtain defect-free, crack-free and uniform $\mathrm{TiO}_{2}$ selective layer, most of reported fabrication methods adopted tedious and costly multiple-coating strategies $[3,17,18]$. A common problem associated with current fabrication methods is the blockage of the pores of the porous membrane substrate, which can lead to a dramatic reduction in flux $[3,5]$. To 
solve the problem, intermediate layers must be formed with larger size $\mathrm{TiO}_{2}$ precursor particles before the desirable size $\mathrm{TiO}_{2}$ particle can be coated $[3,5]$. Though these measures have significantly reduced the blockage problem, the flux of the resultant membranes is low, attributing to the inherent disadvantages of random particle packing and the multi-intermediate layer structures [17, 18]. Introducing the intermediate layers unavoidably increases thickness, leading to a decrease in flux. Low porosity (i.e., $<36 \%$ ) is another inherent disadvantage of randomly packed particle structures, due to the presence of dead-ended pores that make no contribution to the membrane permeability $[17,18]$.

In order to overcome the intrinsic disadvantages of the randomly packed particle structures, we and others recently demonstrated a new dense layer $\mathrm{TiO}_{2}$ structure formed by randomly packed nanofibers via filtration and spin-coating methods $[6,17,18]$. Such a dense layer structure has distinctive advantages over the randomly packed particle structures. It possesses high porosity ( $>70 \%)$ [17] and more importantly, no dead-ended pores present in the porous structure formed by such one dimensional nanofibers [17]. However, the method has disadvantages. The nanofibers used for the membrane fabrication need to be hydrothermally synthesized, and a number of purification and treatment steps must be taken before use. To avoid the blockage problem, it must either use smaller pore size supporting substrate or adopt a multi-intermediate layer approach. The pore size and porosity of the resultant membrane prepared in such a way is almost solely determined by the dimensional parameters of the precursor nanofibers. Nanofibers with different dimensional parameters, especially the diameters of the fiber, must be purposely synthesized in order to effectively alert the pore size and porosity of the resultant membrane. In addition, controlling thickness and uniformity of the coating layer are found to be difficult, especially when the coating area is large. Furthermore, the resultant coating layer possesses relatively weak mechanical strength and adhesion between the coating layer and the porous membrane substrate.

In this work, we fabricated a high performance inorganic filtration membrane by directly growing titanate nanotube separation layer on a porous titanium membrane substrate. The resultant titanate nanotube membrane retains superior transport/separation properties of a one-dimensional nanofiber 
based membrane structure suitable for removal waterborne pathogens. In our work, E. coli is selected as test bacteria to demonstrate the applicability.

\section{Experimental}

\subsection{Titanate nanotube membrane fabrication}

Titanium porous substrate membrane disks with $30 \mathrm{~mm}$ in diameter, $2 \mathrm{~mm}$ thickness and average pore size of $1.0 \mu \mathrm{m}$ were custom-manufactured by Shijiazhuang BEOT Co. LTD, P.R. China. Titanate nanotube membrane (TNM) was fabricated by direct growing titanate nanotubes onto a porous titanium membrane substrate using a pre-prepared titanate seed solution under hydrothermal condition. For our method, an apposite titanate seed solution is essential for obtaining the desirable nanotube membrane structure. The titanate seed solution was synthesized by hydrothermal treatment of metal titanium foil $(2.0 \mathrm{~g})$ in $10 \mathrm{M} \mathrm{NaOH}$ solution at $150^{\circ} \mathrm{C}$ for $2 \mathrm{~h}$ and was then ultrasonically treated for 10 min to achieve homogeneous dispersion. The resultant seed solution contains mainly the titanate floccules (Fig. 1A) that gives a milky-turbid appearance (Fig. 1B) distinctively different to the initial transparent $\mathrm{NaOH}$ solution (Fig. 1C). These titanate floccules act as precursors/seeds to facilitate the formation of dense titanate nanotubes under hydrothermal conditions [19]. The formation of the TNM was carried out by adding $2 \mathrm{~mL}$ seed solution to the top of the porous titanium membrane substrate assembled in a special holder placed in an autoclave at $150^{\circ} \mathrm{C}$ for $5 \mathrm{~h}$. The resultant nanotube membrane was adequately rinsed with $0.1 \mathrm{M} \mathrm{HCl}$ and deionized water. The as-prepared TNM was subsequently calcined at $500^{\circ} \mathrm{C}$ for $2 \mathrm{~h}$ to improve the mechanical strength. It should be noted that in addition to titanate seeds, titanium membrane substrate is also a main titanium source for the titanate nanotube growth.

\subsection{Titanate nanotube membrane performance}

The separation performance of the obtained TNM were evaluated by filtering $100 \mathrm{~mL}$ of dilute polystyrene (PS) microspheres (or E. coli) solutions under a vacuum of 0.2 bar. PS microspheres of 50, 100, 200 and $500 \mathrm{~nm}$ were purchased from Alfa Aesar, and 0.005 wt.\% solutions were prepared by dispersing PS microspheres in high-purity deionized water (Millipore Corp., $18 \mathrm{M} \Omega \mathrm{cm}$ ). E. coli were 
incubated in $\mathrm{LB}$ nutrient solution at $37^{\circ} \mathrm{C}$ on a rotary shaker at $150 \mathrm{rpm}$ for $24 \mathrm{~h}$ followed by a three repetitive washing process with a sterile physiological saline solution via centrifuging for $10 \mathrm{~min}$ at $5000 \mathrm{rpm}$. The resultant E. coli suspension was diluted to $\sim 4 \times 10^{6} \mathrm{CFU} \mathrm{mL}^{-1}$ with a sterile physiological saline solution and used as the feed for filtration experiments. The concentrations of PS microspheres in the feed and permeated solution were measured by UV-vis spectroscopy [17, 18]. The concentrations of $E$. coli in the feed and permeated solution were determined using the standard plate count method and double confirmed with the BacLight ${ }^{\mathrm{TM}}$ kit fluorescent microscopic method (Olympus, DS-5Mc, Japan) [20, 21]. The retention $(R)$ of the TNM was determined using the equation (1):

$$
R \quad(\%) \quad=\quad\left(1-C_{\text {permeate }} / C_{\text {feed }}\right) \quad \times \quad 100
$$

where $C_{\text {permeate }}$ and $C_{\text {feed }}$ are the concentrations of PS microspheres or E. coli permeate collected and the feed, respectively.

\subsection{Sample characterization}

SEM and TEM images of the samples were obtained using a JEOL JSM-6300F field emission scanning electron microscopy and FEI Tecnai 20 transmission electron microscopy, respectively. XRD patterns were obtained using a Shimadzu XRD-6000 diffractometer, equipped with a graphite monochromator. $\mathrm{Cu} \mathrm{K \alpha}$ radiation $(\lambda=1.5418 \AA)$ and a fixed power source $(40 \mathrm{kV}$ and $40 \mathrm{~mA})$ were used. For each sample (feed and permeated fluid), the UV-vis spectroscopy was recorded from 190-800 nm using a Shimadzu (Tokyo, Japan) UV 1691 spectrophotometer. The absorbance at 225 nm was used to determine the concentration of PS microspheres solution. The E. coli SEM samples were prepared using the E. coli retained on the TNM. The TNM with retained E. coli was pretreated with $3 \%$ glutaraldehyde for $1 \mathrm{~h}$ at room temperature and washed with $0.1 \mathrm{M}$ cacodylate buffer $(\mathrm{pH} 7.4)$ for 10 min to immobilize the retained $E$. coli onto the TNM. The sample was then subjected to a 20 min post treatment process with $1 \%$ osmium tetraoxide followed by a dehydration process using a graded series of ethanol (70, 90 and 100\%) and 100\% amyl acetate 2 times, each for $10 \mathrm{~min}$, respectively. The 
samples were then dried using Denton Vacuum critical point dryer (Denton Vacuum, Inc. USA), and ready for SEM characterization.

\section{Results and discussion}

\subsection{Construction of titanate nanotube separation layer}

Fig. 2A schematically illustrates TNM fabrication process. The porous titanium membrane substrate has a mean pore size of $1.0 \mu \mathrm{m}$ and porosity of $25 \%$ (Fig. 2B). After hydrothermal reaction, the hierarchical titanate nanotube structure was shown in Fig. 2C. The as-prepared TNM was subsequently calcined at $500^{\circ} \mathrm{C}$ for $2 \mathrm{~h}$ to improve the mechanical strength. Fig. 2D shows SEM image of typical surface morphology of the annealed TNM. Such morphology was found to cover the entire coating

surface $\left(3.1 \mathrm{~cm}^{2}\right)$. The average length of the titanate nanotubes was found to be in a sub-millimeter range. XRD patterns suggested that the composition of the nanotube layer after hydrothermal reaction was titanate (curve a in Fig. 3). After calcination, the obtained XRD data confirmed that there was no phase transformation occurred and the titanate was the domination composition of the annealed nanotubes, attributing to the high sodium content in the resultant titanate nanotubes (curve b in Fig. 3) $[22,23]$. A detailed examination revealed that the formed titanate nanotube layer possessed a hierarchical structure with a $0.35 \mu \mathrm{m}$ dense nanotubular surface layer interconnected to a $2.5 \mu \mathrm{m}$ intermediate nanotubular layer. It is to note that an initial attempt to fabricate the titanate nanotube layer by directly immersing the porous titanium membrane in $10 \mathrm{M} \mathrm{NaOH}$ solution under various hydrothermal conditions was failed due to the blockage of the titanium substrate pores that caused by the formation of dense titanate particle layer within the pores, and between the substrate and nanotube structures [24-26]. It is also to note that comparison experiments were performed using the procedure shown in Fig. 2A excepting that the seed solution was replaced by $2 \mathrm{~mL}$ of $10 \mathrm{M} \mathrm{NaOH}$ solution. The resultant titanate porous membrane revealed a 'bird-nest' structure with pore sizes up to $700 \mathrm{~nm}$ (Fig. 4).

\subsection{Morphological and structural characteristics}


Fig. 5A displays a high magnification SEM image of TNM surface after calcination. It revealed that the top dense nanotube layer responsible for membrane selectivity was formed by the directly grown interwoven nanotubes. Such a network formed by the ultra-long interwoven nanotubes possesses strong mechanical strength and enables a uniform coverage to produce crack-free and pinhole-free membrane structure. The structural parameters of individual nanotube were investigated by high resolution TEM (see the insert in Fig. 5A). The internal and outer diameters of the nanotube were found to be about 6 $\mathrm{nm}$ and $12 \mathrm{~nm}$, respectively. The thickness of the nanotube wall was approximately $3 \mathrm{~nm}$, and the measured interlayer spacing was about $7.8 \AA$. These parameters are similar to those obtained via a normal hydrothermal method [27]. In addition, the cross-section SEM image shown in Fig. 5B demonstrates a well preserved hierarchical nanotube structure after calcination. A further investigation revealed that such a hierarchical nanotube structure can be perfectly preserved when the calcination temperature was below $500^{\circ} \mathrm{C}$. A calcination temperature of $550^{\circ} \mathrm{C}$ led to a partially collapsed nanotube structure. The nanotube structure was found to be completely destroyed when the temperature was greater than $650^{\circ} \mathrm{C}$.

With the randomly packed nanofibers structure, the pore size and porosity are determined by the diameter of the precursor nanofibers $[17,18]$. The pore size equals the precursor nanofibers' diameter would be the smallest achievable pore size for the randomly packed nanofibers structure. In this regard, the directly grown nanotube structure is advantageous. This is because the pore size and porosity of the network structure formed by interweaving smaller diameter nanotubes (i.e., $\sim 12 \mathrm{~nm}$ ) can be tuned by controlling the density and thickness of the top layer. The pore size distribution of the TNM was found to be ranged from 10 to $110 \mathrm{~nm}$, determined by a liquid-liquid displacement method [28]. The obtained data also revealed that over $80 \%$ of pores were within the range of 25 to $50 \mathrm{~nm}$ while only less than $0.5 \%$ of pores having sizes greater than $100 \mathrm{~nm}$. For this reason, we define the obtained TNM as a microfiltration membrane although over $90 \%$ of pores were within the ultrafiltration membrane pore size range. A membrane with such a pore size distribution can be used for a wide range of applications, particularly, for removal of waterborne pathogens and viruses [29-34]. 


\subsection{Transport and separation properties}

The permeability and separation performance of the TNM were evaluated. A steady pure water permeability of $680 \pm 28 \mathrm{~L} \mathrm{~m}^{-2} \mathrm{~h}^{-1}$ bar $^{-1}$ was obtained. The separation characteristics were evaluated by filtering $100 \mathrm{~mL}$ water dispersed with 0.005 wt.\% of polystyrene (PS) microspheres under a constant applied pressure of 0.2 bar. Fig. 6A shows the effect of PS microsphere size on the steady permeability and the percentage retention. The steady permeability obtained from all microsphere sizes investigated were within $615 \pm 25 \mathrm{~L} \mathrm{~m}^{-2} \mathrm{~h}^{-1}$ bar $^{-1}$, suggesting the size of the PS microsphere has an insignificant influence on the membrane permeability. A near $100 \%$ retention was achieved for all microsphere sizes investigated (50,100, 200 and $500 \mathrm{~nm}$ in diameter). The lowest retention obtained from filtrating $50 \mathrm{~nm}$ microspheres was as high as $97 \%$. These results demonstrate that the TNM has the ability to filter out particles with sizes greater than $50 \mathrm{~nm}$. The strong mechanical strength is one of advantages of inorganic membranes. The TNM fabricated in this work utilizes a porous titanium membrane substrate (30 $\mathrm{mm}$ in diameter and $2.0 \mathrm{~mm}$ in thickness) fabricated by compressing titanium powder under high pressure and temperature. The mechanical strength of the substrate is sufficient to sustain the pressure required to drive a microfiltration or an ultrafiltration process. Nevertheless, the mechanical properties of the membrane are also largely determined by the mechanical properties of the nanotubular layer and the adhesion between the two parts. The directly grown titanate nanotubes possess strong adhesion to the porous substrate. The entire nanotube network (separation layer) was formed by the interwoven ultra-long nanotubes network directly grown to the substrate. The high temperature calcination further enhances the mechanical strength of the nanotube network and their adhesion to the substrate. Because of these, the resultant TNM possesses strong mechanical strength. The membrane regeneration is an important practical issue. The superior mechanical properties of the TNM can be utilized to regenerate membrane by a means of back-flushing. In this work, the performance of the regenerated TNM was evaluated using 0.005 wt.\% of $50 \mathrm{~nm}$ PS microspheres. The steady permeability was measured during each filtration cycle and a cycle was terminated when the measured permeability decreased to $50 \%$ of its steady permeability. The filtrated volume was measured and equal volume of water was added back 
to the feed. The membrane was then regenerated by back-flushing compressed air under a controlled pressure of 0.5 bar for $2 \mathrm{~min}$. The retained microspheres by the membrane were re-dispersed back to the feed by the back-flushed air. The concentration of microspheres in the feed should be equal to their original concentration if all retained microspheres were re-dispersed during the back-flushed regeneration process. There should not be any significant change in the steady permeability and the percentage retention of consecutive filtration cycles if the back-flushing regeneration method is effective. Fig. 6B shows the membrane performance obtained from 5 consecutive back-flushing regeneration cycles. A near $98 \%$ retention was observed from all cycles, implying that there was no structural damage occurred, as the result of back-flushing actions. More importantly, no significant decrease was observed from the steady permeability of consecutive cycles, indicating the membrane can be effectively regenerated by the simple procedure of back-flushing air. The retention of PS microspheres by the TNM was characterized using SEM technique. A mixture of PS microspheres was prepared by mixing the original 0.005 wt.\% PS microsphere dispersions of 50, 100 and $200 \mathrm{~nm}$ in a ratio of 1:1:1 (v/v/v). Fig. $6 \mathrm{C}$ and D show the surface and cross section SEM images of the TNM after filtration, respectively. It can be seen that different sizes PS microspheres were retained by the dense nanotube layer (Fig. 6C) and no microsphere was able to penetrate through the dense nanotube layer (Fig. 6D). These observations demonstrate the superior structural properties of the TNM.

For comparison purpose, the titanate porous membrane (Fig. 4) fabricated using $2 \mathrm{~mL}$ of $10 \mathrm{M} \mathrm{NaOH}$ solution rather than seed solution was examined by filtrating different sizes PS microsphere dispersions. Retentions of $42 \%, 13 \%, 6.3 \%$, and $0.5 \%$ were obtained for PS microspheres of $500 \mathrm{~nm}, 200 \mathrm{~nm}, 100$ nm, and $50 \mathrm{~nm}$, respectively (Fig. 7A). Except for $50 \mathrm{~nm}$ PS microspheres, a higher permeability of approximate $1000 \mathrm{~L} \mathrm{~m}^{-2} \mathrm{~h}^{-1} \mathrm{bar}^{-1}$ was obtained for all other PS microsphere sizes (Fig. 7A). Higher flux $\left(\sim 1130 \mathrm{~L} \mathrm{~m}^{-2} \mathrm{~h}^{-1} \mathrm{bar}^{-1}\right)$ obtained from $50 \mathrm{~nm}$ microsphere can be attributed to the larger pore size (Fig. 4) that allows $50 \mathrm{~nm}$ PS microsphere directly filtrating through the membrane without the pores being blocked. The lower fluxes obtained from PS microsphere sizes greater than $100 \mathrm{~nm}$ may be due to the blockage of pores as a result of PS microspheres retained inside of the pores. Cross-section SEM image 
shown in Fig. 7B revealed that the PS microspheres with 50, 100 and $200 \mathrm{~nm}$ in diameter can be found inside of the pores. These results further confirm that the titanate seed solution plays an important role in formation of the dense titanate nanotube layer with high selectivity.

\subsection{E. Coli removal}

Serious global fresh water shortage problems force us to recycle/reuse water. Effective removal of biohazards (e.g., waterborne pathogens) from treated water is one of the most important aspects to safeguard water recycle/reuse. Owing to their advantageous properties, the inorganic membranes could play an important role in the field of purified recycled water. The applicability of the TNM for removal of waterborne pathogens was therefore investigated using E. coli as a test species. All filtration experiments were performed under a constant pressure of 0.2 bar using a feed containing $4 \times 10^{6}$ $\mathrm{CFUmL}^{-1}$ E. coli. Fig. $8 \mathrm{~A}$ shows the membrane performance characteristics. The membrane permeability decreased initially with time and reached a near steady permeability of $608 \mathrm{~L} \mathrm{~m}^{-2} \mathrm{~h}^{-1} \mathrm{bar}^{-1}$ after 40 min of filtration. Complete E. coli removal (i.e., 100\% retention) was achieved for all cases investigated. For all experiments, the bacterial viability in the filtrated solution was determined using the standard plate count method after $24 \mathrm{~h}$ sample incubation at $37^{\circ} \mathrm{C}$ and were double confirmed with the BacLight ${ }^{\mathrm{TM}}$ kit fluorescent microscopic method [21]. Fig. 8B and $\mathrm{C}$ show the fluorescent microscopic images of viable cells in the feed and the filtrated solution, respectively. The viable cells accumulate Rhodamine 123 appearing as 'green' under fluorescent microscope [21]. It is very obvious that no viable cells were observed in the filtrated solution, suggesting $100 \%$ removal of E. coli was achieved (Fig. 8C). The surface SEM image shown in Fig. 8D suggests that the top dense nanotubular layer was responsible for the retention of $E$. coli. The cross-section SEM image shows no E. coli was able to penetrate through the dense layer (see Fig. 8E).

\section{Conclusions}

In summary, a hierarchically structured titanate nanotube membrane has been successfully fabricated by directly growing nanotubes onto a porous membrane substrate using a seed solution under 
hydrothermal conditions. A hierarchically structured titanate nanotube separation layer constructed by interwoven ultra-long nanotubes directly grown on the substrate possesses superior structural and strong mechanical properties. Benefiting from these properties, TNM is intrinsically protected from cracks and pinholes, and can be readily regenerated via a simple pressure driven back-flushing process. Successful separation of E. coli demonstrates the applicability of TNM for waterborne pathogens removal, which would be of a great interest to the purified recycled water applications. The high selectivity and flux of TNM in addition to its excellent biocompatibility and nontoxic nature make such a membrane highly attractive to medical applications for removal pathogens and other unwanted biological constituents with sizes greater than $50 \mathrm{~nm}$ from highly complex medium.

\section{Acknowledgments}

The authors would like to acknowledge the Australian Research Council for financial support.

\section{References}

[1] J. Coronas, J. Santamaria, Catalytic reactors based on porous ceramic membranes, Catal. Today 51 (3-4) (1999) 377.

[2] A. Julbe, V. Rouessac, J. Durand, A. Ayral, New approaches in the design of ceramic and hybrid membranes, J. Membr. Sci. $316(1+2)(2008) 176$.

[3] L. Zhao, M. Bram, H.P. Buchkremer, D. Stoever, Z. Li, Preparation of $\mathrm{TiO}_{2}$ composite microfiltration membranes by the wet powder spraying method, J. Membr. Sci. 244 (1-2) (2004) 107.

[4] T. Tsuru, T. Kan-no, T. Yoshioka, M. Asaeda, A photocatalytic membrane reactor for VOC decomposition using Pt-modified titanium oxide porous membranes, J. Membr. Sci. $280(1+2)$ (2006) 156.

[5] H. Choi, A.C. Sofranko, D.D. Dionysiou, Nanocrystalline $\mathrm{TiO}_{2}$ photocatalytic membranes with a hierarchical mesoporous multilayer structure: synthesis, characterization, and multifunction, Adv. Funct. Mater. 16 (8) (2006) 1067.

[6] X. Zhang, A.J. Du, P. Lee, D.D. Sun, J.O. Leckie, $\mathrm{TiO}_{2}$ nanowire membrane for concurrent filtration and photocatalytic oxidation of humic acid in water. J. Membr. Sci. $313(1+2)(2008)$ 44.

[7] H. Choi, K. Zhang, D.D. Dionysiou, D.B. Oerther, G.A. Sorial, Effect of permeate flux and tangential flow on membrane fouling for wastewater treatment, Sep. Purif. Technol. 45 (1) (2005) 68.

[8] A. Alem, H. Sarpoolaky, M. Keshmiri, Titania ultrafiltration membrane: Preparation, characterization and photocatalytic activity, J. Eur. Ceram. Soc. 29 (4) (2009) 629.

[9] L.Q. Wu, P. Huang, N. Xu, J. Shi, Effects of sol properties and calcination on the performance of titania tubular membranes, J. Membr. Sci. 173 (2) (2000) 263. 
[10] C. Labbez, P. Fievet, A. Szymczyk, A. Vidonne, A. Foissy, J. Pagetti, Analysis of the salt retention of a titania membrane using the "DSPM" model: effect of $\mathrm{pH}$, salt concentration and nature, J. Membr. Sci. 208 (1-2) (2002) 315.

[11] M.A. Anderson, M.J. Gieselmann, Q. Xu, Titania and alumina ceramic membranes, J. Membr. Sci. 39 (3) (1988) 243.

[12] Z. Li, N. Qiu, G. Yang, Effects of synthesis parameters on the microstructure and phase structure of porous 316L stainless steel supported $\mathrm{TiO}_{2}$ membranes, J. Membr. Sci. 326 (2) (2009) 533.

[13] S.P. Albu, A. Ghicov, J.M. Macak, R. Hahn, P. Schmuki, Self-organized, free-standing $\mathrm{TiO}_{2}$ nanotube membrane for flow-through photocatalytic applications, Nano Lett. 7 (5) (2007) 1286.

[14] X.W. Zhang, A.J.H. Du, P.F. Lee, D.D. Sun, J.O. Leckie, Grafted multifunctional titanium dioxide nanotube membrane: Separation and photodegradation of aquatic pollutant, Appl. Catal. B: Environ. 84 (1-2) (2008) 262.

[15] Y.M. Sang, Q.B. Gu, T.C. Sun, F.S. Li, C.Z. Liang, Filtration by a novel nanofiber membrane and alumina adsorption to remove copper(II) from groundwater, J. Hazard. Mater. 153 (1-2) (2008) 860.

[16] Y.M. Sang, F.S. Li, Q.B. Gu, C.Z. Liang, J.Q. Chen, Heavy metal-contaminated groundwater treatment by a novel nanofiber membrane, Desalination 223 (1-3) (2008) 349.

[17] X.B. Ke, Z.F. Zheng, H.W. Liu, H.Y. Zhu, X.P. Gao, L.X. Zhang, N.P. Xu, H. Wang, H.J. Zhao, J. Shi, K.R. Ratinac, High-flux ceramic membranes with a nanomesh of metal oxide nanofibers, J. Phys. Chem. B 112 (16) (2008) 5000.

[18] X.B. Ke, H.Y. Zhu, X.P. Gao, J.W. Liu, Z.F. Zheng, High-performance ceramic membranes with a separation layer of metal oxide nanofibers, Adv. Mater. 19 (6) (2007) 785.

[19] A. Kukovecz, M. Hodos, E. Horvath, G. Radnoczi, Z. Konya, I. Kiricsi, Oriented crystal growth model explains the formation of titania nanotubes, J. Phys. Chem. B 109 (38) (2005) 17781.

[20] M.P. Reddy, A. Venugopal, M. Subrahmanyam, Hydroxyapatite-supported Ag-TiO 2 as Escherichia coli disinfection photocatalyst, Water Res. 41 (2) (2007) 379.

[21] M. Berney, F. Hammes, F. Bosshard, H.-U. Weilenmann, T. Egli, Assessment and interpretation of bacterial viability by using the LIVE/DEAD BacLight kit in combination with flow cytometry, Appl. Environ. Microbiol. 73 (10) (2007) 3283.

[22] C.-K. Lee, C.-C. Wang, M.-D. Lyu, L.-C. Juang, S.-S. Liu, S.-H. Hung, Effects of sodium content and calcination temperature on the morphology, structure and photocatalytic activity of nanotubular titanates, J. Colloid Interface Sci. 316 (2) (2007) 562.

[23] E. Morgado, M.A.S. de Abreu, O.R.C. Pravia, B.A. Marinkovic, P.M. Jardim, F.C. Rizzo, A.S. Araujo, A study on the structure and thermal stability of titanate nanotubes as a function of sodium content, Solid State Sci. 8 (8) (2006) 888.

[24] M. Yada, Y. Inoue, M. Uota, T. Torikai, T. Watari, I. Noda, T. Hotokebuchi, Plate, wire, mesh, microsphere, and microtube composed of sodium titanate nanotubes on a titanium metal template, Langmuir 23 (5) (2007) 2815.

[25] X. Peng, A. Chen, Large-scale synthesis and characterization of $\mathrm{TiO}_{2}$-based nanostructures on Ti substrates, Adv. Funct. Mater. 16 (10) (2006) 1355.

[26] M. Miyauchi, H. Tokudome, Super-hydrophilic and transparent thin films of $\mathrm{TiO}_{2}$ nanotube arrays by a hydrothermal reaction, J. Mater. Chem. 17 (20) (2007) 2095.

[27] Z.-Y. Yuan, B.-L. Su, Titanium oxide nanotubes, nanofibers and nanowires, Colloids Surf. A $241(1-3)(2004) 173$.

[28] J.I. Calvo, A. Bottino, G. Capannelli, A. Hernandez, Comparison of liquid-liquid displacement porosimetry and scanning electron microscopy image analysis to characterize ultrafiltration track-etched membranes, J. Membr. Sci. 239 (2) (2004) 189.

[29] S.Y. Yang, I. Ryu, H.Y. Kim, J.K. Kim, S.K. Jang, T.P. Russell, Nanoporous membranes with ultrahigh selectivity and flux for the filtration of viruses, Adv. Mater. 18 (6) (2006) 709.

[30] M.G. McKee, J.M. Layman, M.P. Cashion, T.E. Long, Phospholipid nonwoven electrospun membranes, Science 311 (5759) (2006) 353.

[31] A.G. MacDiarmid, ।"Synthetic metals।": A novel role for organic polymers (Nobel Lecture), 
Angew. Chem. Int. Ed. 40 (14) (2001) 2581.

[32] D. Sun, C. Chang, S. Li, L. Lin, Near-field electrospinning, Nano Lett. 6 (4) (2006) 839.

[33] H. Verweij, Ceramic membranes: Morphology and transport, J. Mater. Sci. 38 (23) (2003) 4677.

[34] R.J. Webby, R.G. Webster, Are we ready for pandemic influenza?, Science 302 (5650) (2003) 1519. 
FIGURE CAPTIONS

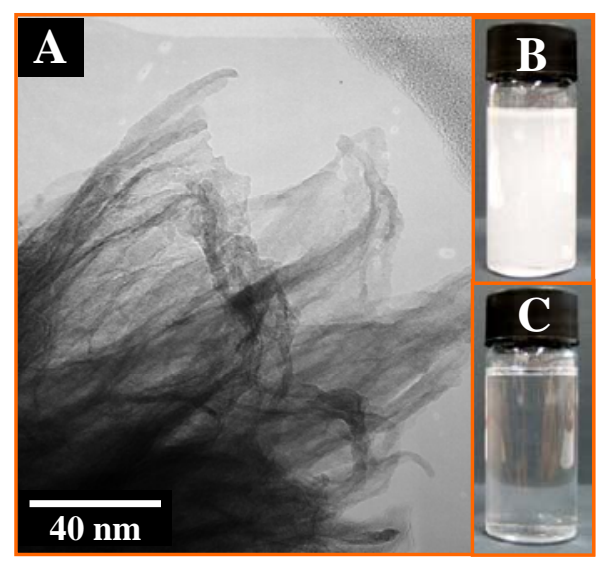

Fig. 1. A) Typical TEM image of titanate seed solution. B) and C) Photos of the resultant seed solution and $10 \mathrm{M} \mathrm{NaOH}$ solution before reaction, respectively. 


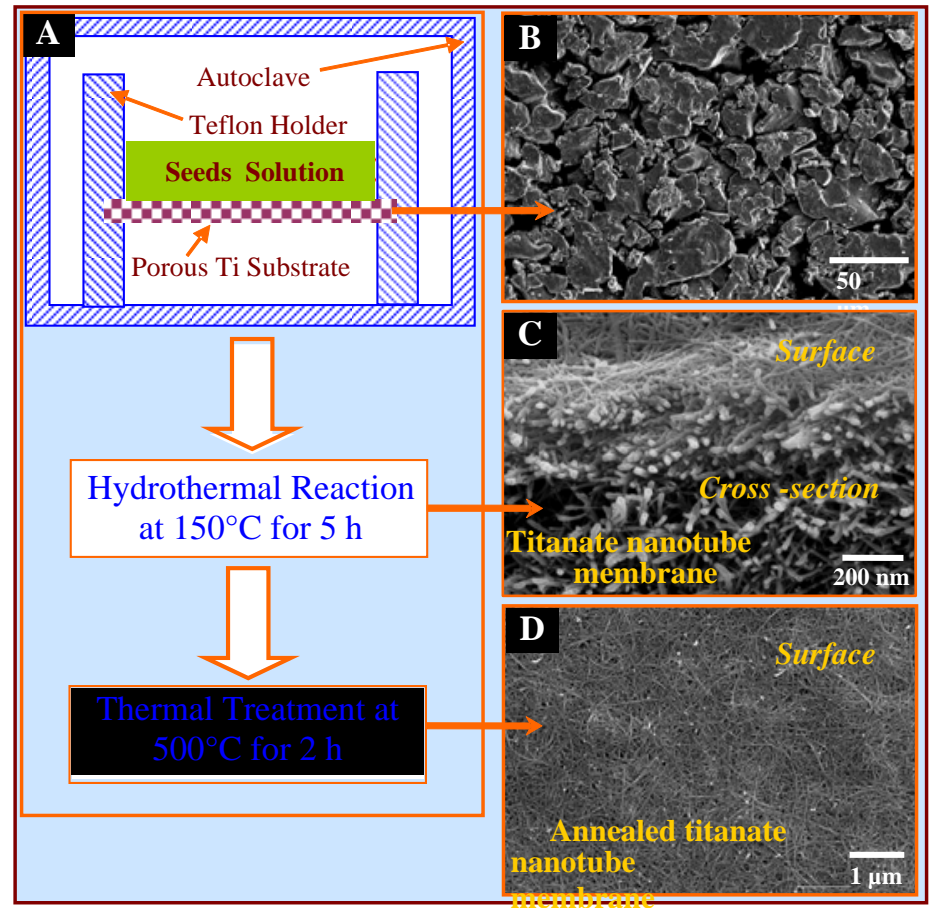

Fig. 2. A) Schematic illustrating a direct hydrothermal growth method for fabrication of TNM. B) Typical SEM image of the porous titanium membrane substrate. C) Typical SEM image of an assynthesized TNM. D) Typical SEM image of an annealed TNM. 


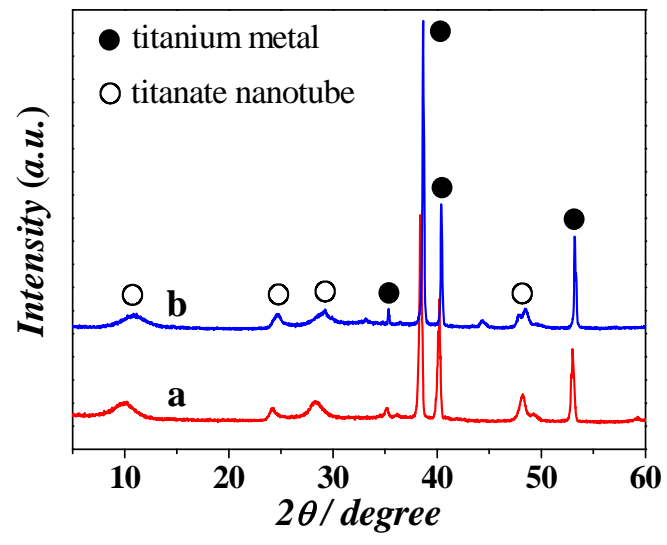

Fig. 3. X-ray diffraction patterns of the as-synthesized TNM (curve a), and the TNM annealed at $500^{\circ} \mathrm{C}$ for $2 \mathrm{~h}$ (curve b). 

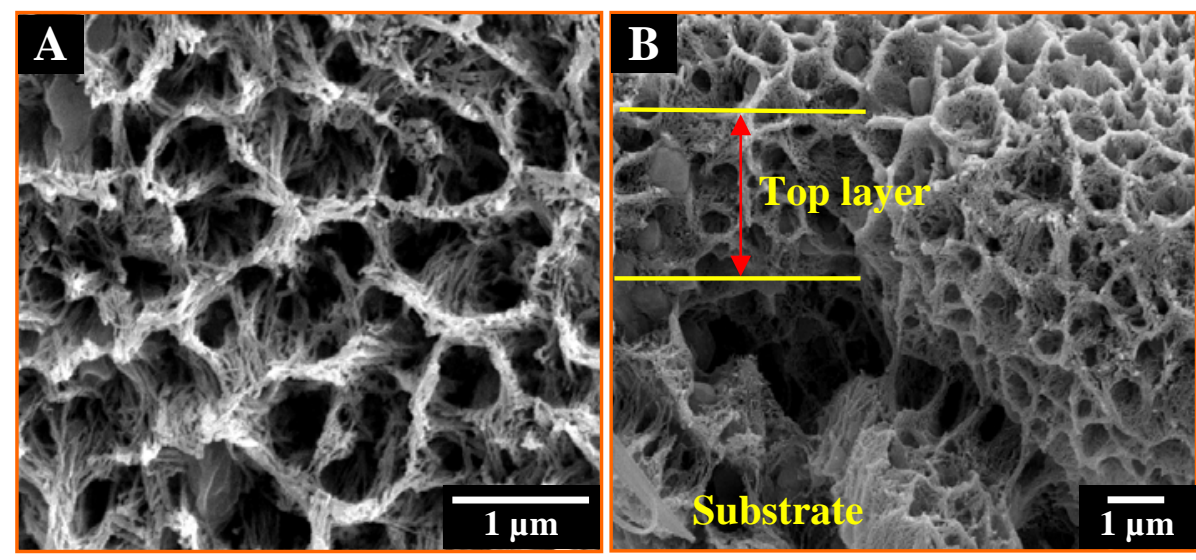

Fig. 4. Surface (A) and cross-section (B) SEM images of the titanate porous membrane hydrothermally fabricated using $2 \mathrm{~mL}$ of $10 \mathrm{M} \mathrm{NaOH}$ solution at $150^{\circ} \mathrm{C}$ for $5 \mathrm{~h}$ and then annealed at $500^{\circ} \mathrm{C}$ for $2 \mathrm{~h}$. 

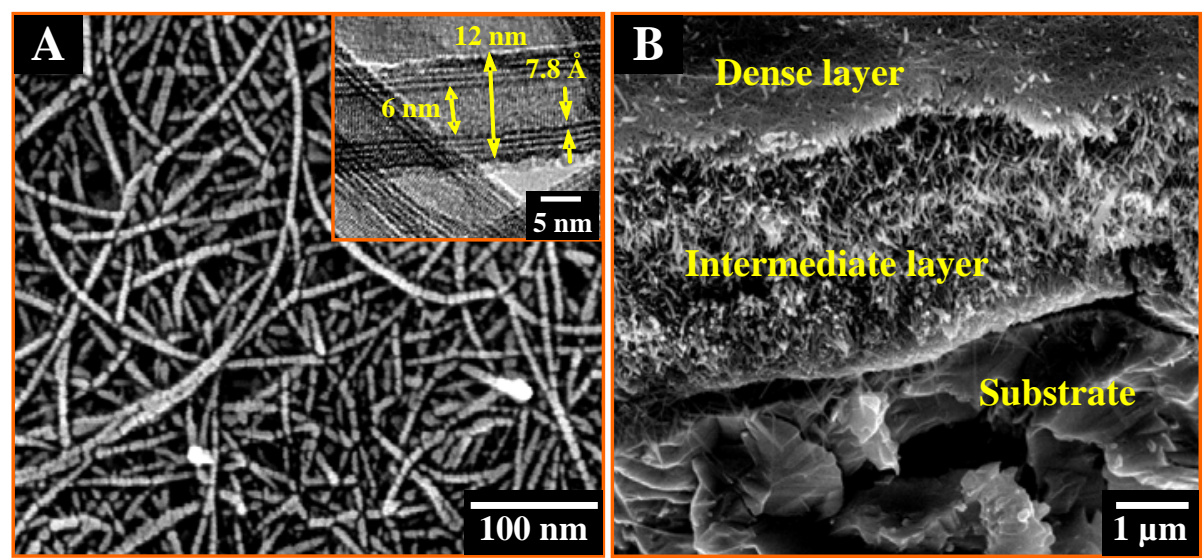

Fig. 5. SEM images of the resultant TNM annealed at $500^{\circ} \mathrm{C}$ for $2 \mathrm{~h}$. A) A typical surface SEM image; the inset is a TEM image of individual titanate nanotube. B) A typical cross-section SEM image. 

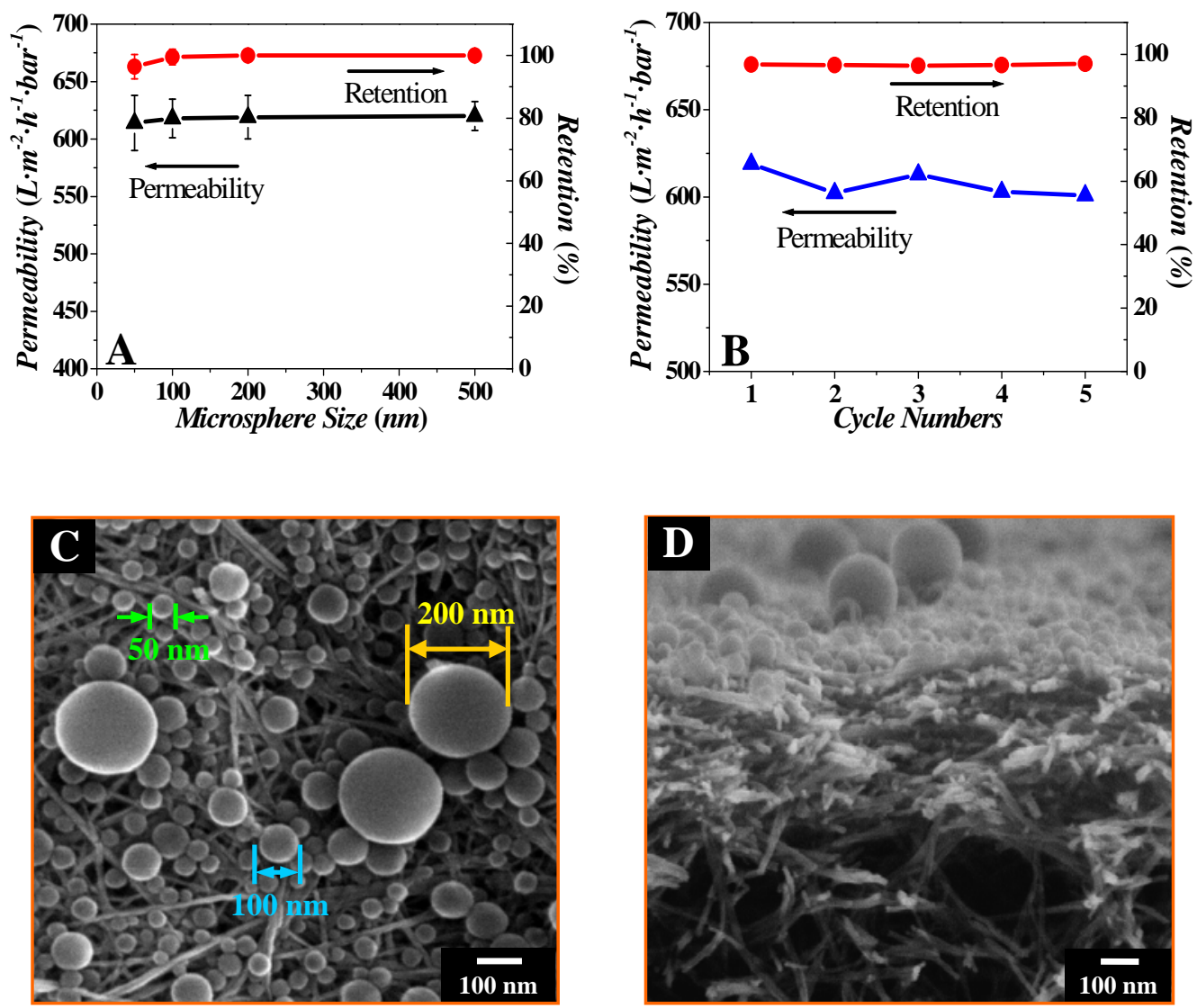

Fig. 6. A) Effect of PS microsphere size on permeability and retention of TNM. B) TNM filtration performance of $50 \mathrm{~nm}$ PS microspheres over five consecutive back-flushing regeneration cycles. C) SEM image of retained PS microspheres on TNM surface. D) A cross-section SEM image of the TNM after filtration of PS microspheres mixture. 

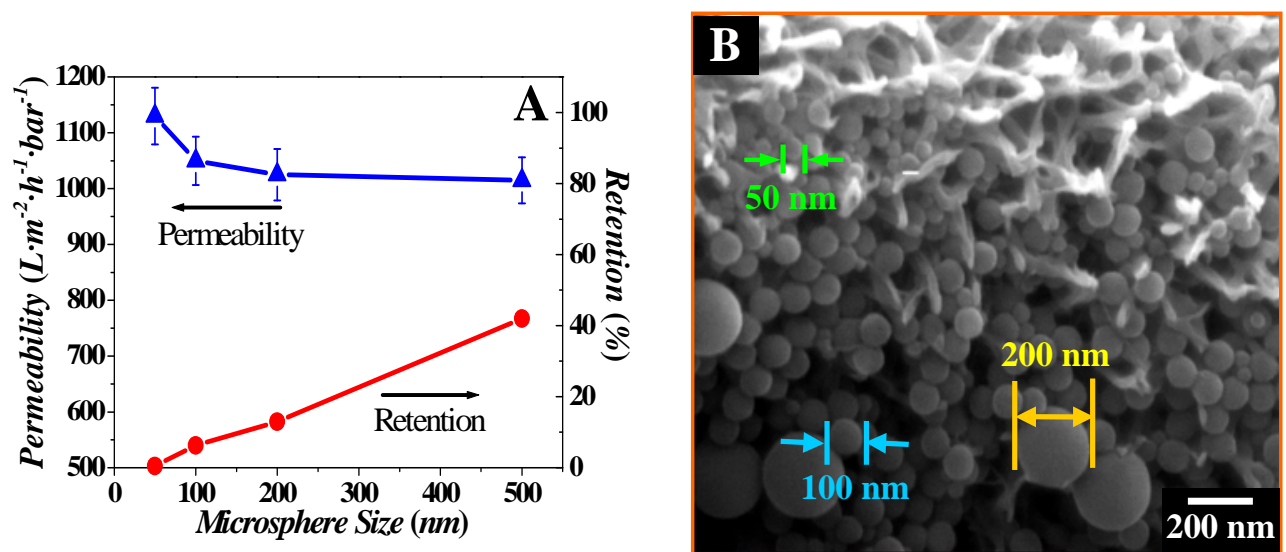

Fig. 7. A) Filtration properties of the titanate porous membrane tested with a 0.005 wt.\% PS microspheres feed containing different sizes PS microspheres of 50, 100, 200 and $500 \mathrm{~nm}$ in diameter. B) A cross-section SEM image of the titanate porous membrane after filtration of PS microspheres mixture. 

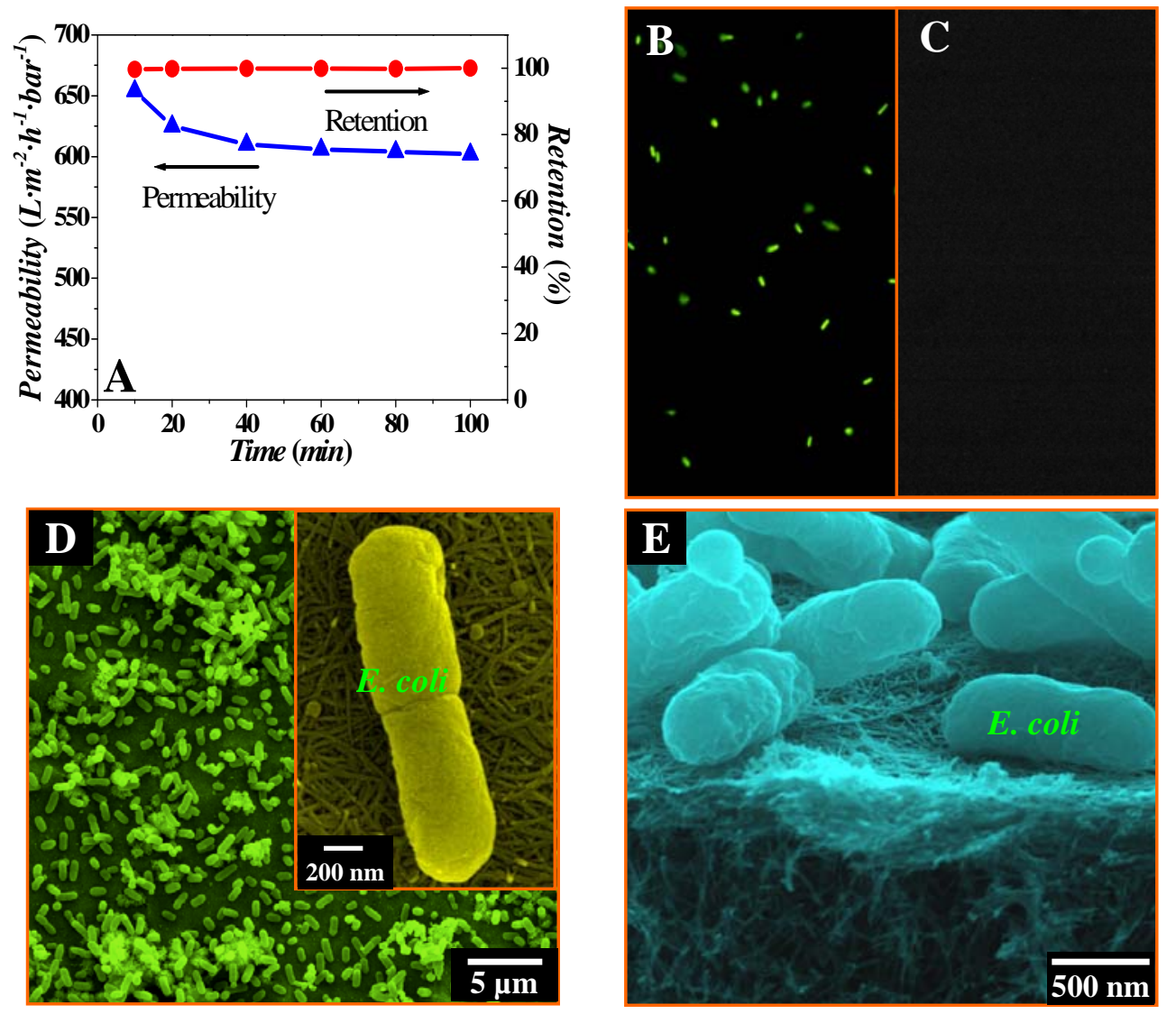

Fig. 8. A) TNM filtration performance of E. coli with an initial concentration of $\sim 4 \times 10^{6} \mathrm{CFUmL}^{-1}$. B) and C) The fluorescent microscopic images of E. coli feed and permeate, respectively. D) A SEM image of retained E. coli on the TNM after filtration (low magnification) and a high magnification SEM image (inset). E) A cross-section SEM image of TNM after filtration of E. coli. 